\title{
Motion Tracking and Strain Map Computation for Quasi-Static Magnetic Resonance Elastography
}

\author{
Y.B. Fu ${ }^{1, *}$, C.K. Chui ${ }^{1}$, C.L. Teo ${ }^{1}$, and E. Kobayashi ${ }^{2}$ \\ ${ }^{1}$ Department of Mechanical Engineering, National University of Singapore, Singapore \\ ${ }^{2}$ Department of Precision Engineering, The University of Tokyo, Tokyo, Japan \\ g0800248@nus.edu.sg
}

\begin{abstract}
This paper presents a new imaging method for quasi-static magnetic resonance elastography (MRE). Tagged magnetic resonance (MR) imaging of human lower leg was acquired with probe indentation using a MR-compatible actuation system. Indentation force was recorded for soft tissue elasticity reconstruction. Motion tracking and strain map of human lower leg are calculated using a harmonic phase (HARP)-based method. Simulated tagged MR images were constructed and analyzed to validate the HARP-based method. Our results show that the proposed imaging method can be used to generate accurate motion distribution and strain maps of the targeted soft tissue.
\end{abstract}

Keywords: MR tagging, HARP, motion tracking, strain, soft tissue indentation.

\section{Introduction}

Elasticity is an important property for characterization of biological soft tissues. Local changes in mechanical properties of soft tissues may indicate the presence of tumors and other diseases [1]. In order to quantitatively study the soft tissue mechanical properties, Magnetic Resonance Elastography (MRE) has been developed in recent years. MRE can be categorized into dynamic MRE [2-4] and static or quasi-static MRE [5,6]. However, there are less literature about the quasi-static MRE due to the difficulty of motion tracking and strain calculation of soft tissue.

Various techniques, such as Ultrasound, Computed Tomography (CT), and Magnetic Resonance Imaging (MRI) have been used to image the spatial deformation of soft tissue. Tagged MRI is widely used for the quantification of motion and deformation of cardiac tissue due to its easy access and good performance. However, the applicability of Tagged MRI on other parts of human body has not been investigated. In this paper, we extended the use of tagged MRI technique to other biological soft tissue by building a MR compatible actuating device synchronized with simulated electrocardiogram (ECG) signal. Large indentation tests on human lower leg were conducted using this imaging method.

Template Matching [7-10], Active Geometry [11-13], Optical Flow [14-17] and Harmonic Phase (HARP) [18] are the main methods for tagged MR images processing. Template matching methods calculate the displacement by tracking the

\footnotetext{
* Corresponding author.
} 
tag lines. Optical flow is advantageous over template matching in providing a dense estimation of the motion field instead of a sparse set of data located at the tag lines. However, the optical flow methods require a material point with constant brightness which is not available for tagged MR images due to tag fading. The HARP method proposed by Osman et al. [20] is widely spread in cardiac image processing [21,23]. However, the applicability of HARP analysis on soft tissue other than cardiac tissue has not been investigated. In this paper, the applicability and accuracy of HARP analysis for automatic motion tracking and strain calculation was investigated on tagged MR images of human lower leg.

\section{Materials and Methods}

\subsection{MR Imaging}

A MR-Compatible actuation system is developed for tagged MR imaging of soft tissue indentation (Fig. 1(a)). ECG signal is simulated and outputted to both the motor controller and MR scanner. MRI k-space data acquisition is synchronized with the simulated ECG signal. Specific k-space segment data is acquired and filled into corresponding images repeatedly after each ECG period. Twenty images are acquired over multiple ECG periods within time duration of 10-20s with GE SIGNA 1.5T MRI Scanner. Ultrasonic motor is controlled by microprocessor based controller interfaced with USB-6221 DAQ device. The control software and signal processing is implemented using LabView version 8.6. Motor is activated to rotate when $R$ peak of simulated ECG is detected and stop after one full rotation. Indentation of the actuation device is synchronized with the simulated ECG signal. This ensures the deformation of imaged object to be consistent during each ECG period.

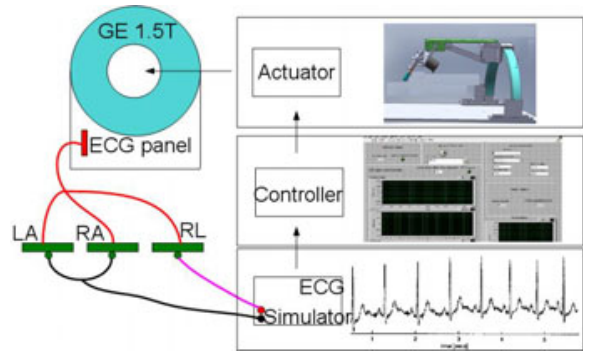

a

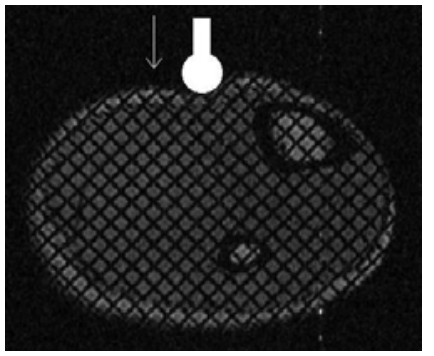

b

Fig. 1. a: Overview of imaging system, b: Human lower leg indentation diagram

Indentation experiment was conducted on a volunteer's lower leg. Indentation force was recorded by a force sensor. The lower leg was indented at the top middle region of the leg using a sphere-shaped indenter with a diameter of $15 \mathrm{~mm}$ (Fig. 1(b).). MR tags were generated by applying the tagging sequence immediately after the detection of the R-wave. Gradient-echo images were acquired during the entire imaging cycle to capture the displacement of the tags. Tagging period of $5 \mathrm{~mm}$ is used. 
Other imaging parameters are as follows: flip angle, $15^{\circ}$; tag orientation, $45^{\circ}$; echo time (TE), $1.704 \mathrm{msec}$; repetition time (TR), $6.032 \mathrm{msec}$; slice thickness, $15 \mathrm{~mm}$; spacing between slices, $15 \mathrm{~mm}$; field of view (FOV), $20 \times 20 \mathrm{~cm}$. The first and the seventh frames of the tagged images are shown in Fig. 1(b) and Fig. 4(a).

\subsection{HARP Analysis}

The spatial modulation of magnetization (SPAMM) sequence modulates the longitudinal magnetization in a sinusoidal manner, which produces an array of spectral peaks (harmonic peaks) in the Fourier domain (k-space). A circular band-pass filter (Fig. 4(b)) is often used to extract the off-center harmonic peaks. Harmonic phase image can be calculated by applying the inverse Fourier transform of a harmonic peak. Since the harmonic image is complex, it has both magnitude and phase at each pixel. The magnitude image reflects the anatomy of the lower leg and is used for segmentation of the leg in this study. The harmonic phase angle of the leg can be used to infer the tissue material property and remains invariant through the image sequences [19]. Displacement distribution and strain maps are calculated from these harmonic phase images.

Motion Tracking. The phase of the image at time $t$ is given by

$$
\emptyset(\boldsymbol{y}, t)=\boldsymbol{w}^{T} \boldsymbol{x}(\boldsymbol{y}, t)
$$

where $\varnothing(\boldsymbol{y}, t)$ is the phase, $\boldsymbol{w}$ is the frequency vector of a harmonic spectral peak, $\boldsymbol{x}$ is the spatial position, $\boldsymbol{y}$ is the image coordinate [20]. At time $t$, the phase shift can be calculated as

$$
\varnothing(y, t)-\emptyset(y, 0)=w^{T}(x(y, t)-x(y, 0))=w^{T} u(y)
$$

where $\boldsymbol{u}(\boldsymbol{y})$ is the displacement vector. Since the phase $\varnothing(\boldsymbol{y}, t)$ differs from the harmonic phase angle $a(\boldsymbol{y}, t)$ by a multiple of $2 \pi$,

$$
\Delta a=\operatorname{wrap}(a(\boldsymbol{y}, t)-a(\boldsymbol{y}, 0))=\operatorname{wrap}\left(\boldsymbol{w}^{T} \boldsymbol{u}(\boldsymbol{y})\right)
$$

where the nonlinear wrapping function is given by

$$
\operatorname{wrap}(\varnothing)=\bmod (\varnothing+\pi, 2 \pi)-\pi .
$$

If $\left|\boldsymbol{w}^{T} \boldsymbol{u}(\boldsymbol{y})\right|<\pi, \boldsymbol{u}(\boldsymbol{y})$ can be calculated by

$$
\boldsymbol{u}(\boldsymbol{y})=\left(\boldsymbol{w}^{T}\right)^{-1} \Delta a
$$

If $\left|\boldsymbol{w}^{T} \boldsymbol{u}(\boldsymbol{y})\right|>\pi, \Delta a$ is always wrapped. For soft tissue indentation, it is reasonable to assume that the displacement varies smoothly within the soft tissue. Given this assumption, $\boldsymbol{w}^{T} \boldsymbol{u}(\boldsymbol{y})$ can be calculated by unwrapping $\Delta a$ starting from a pixel where $\left|\boldsymbol{w}^{T} \boldsymbol{u}(\boldsymbol{y})\right|<\pi$ is satisfied. In this study, a quality guided phase unwrapping method is used.

Strain Calculation. Once the displacement field $\boldsymbol{u}(\boldsymbol{y})$ is calculated, the deformation gradient tensor of the lower leg is given by

$$
F=\nabla_{\mathbf{y}} u(y)+I
$$


Therefore the 2D Lagrangian strain tensor can be calculated as

$$
E=\frac{1}{2}\left(F^{T} F-I\right)
$$

However, this method does not perform well in the presence of noise since the phase unwrapping procedure is needed to calculate $\boldsymbol{u}(\boldsymbol{y})$. The strain map is calculated using

$$
\boldsymbol{E}(\boldsymbol{y})=\frac{1}{2}\left(\boldsymbol{H}^{T} \boldsymbol{w}\left(\frac{\partial \emptyset}{\partial y}\right)^{-T} \cdot\left(\frac{\partial \emptyset}{\partial y}\right)^{-1} \boldsymbol{w}^{\mathrm{T}} \boldsymbol{H}-\boldsymbol{I}\right)
$$

where $\boldsymbol{E}(\boldsymbol{y})$ is the 2D Lagrangian strain, $\boldsymbol{H}$ matrices related with the tagging and imaging plane [20,21].

\section{Results}

\subsection{Validation Using Simulated Tagging Images}

Simulated tagging images were used to validate the strain calculation. The simulated tagging images were constructed by applying sinusoidal modulation to a digital circular image. A circular band-pass filter was used to extract the off-center harmonic peak. The radius of the filter was chosen at one third of the tagging frequency. Stretch strains ranging from 0.1 to 0.4 and shear strains ranging from 0.05 to 0.2 were constructed and processed (Fig. 2(a-e)). 100\% Gaussian white noise was added to test the sensitivity of the algorithm to noise. The calculated strains show good agreement with the true values. The error of $E_{x x}$ is calculated to be within $\pm 8.07 \%$ and $\pm 7.21 \%$ for images with and without noise respectively. The error of $E_{x y}$ is within $\pm 14.95 \%$ and $\pm 13.65 \%$ for images with and without noise respectively. The strain calculation is accurate even in the presence of noise (Fig. 3(a-d)).

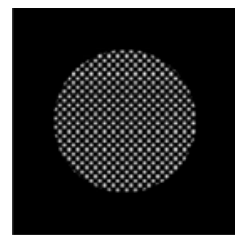

a

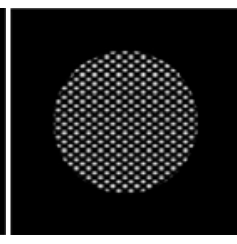

b

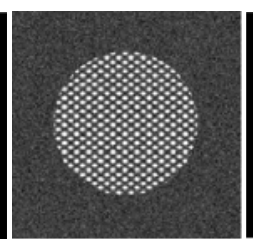

C

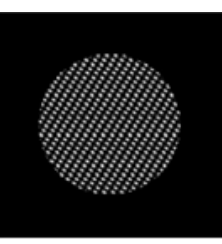

d

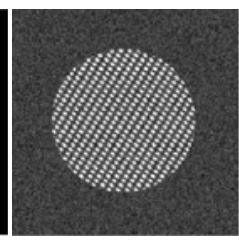

e

Fig. 2. Simulated images. a: Reference image. b: Deformed image of $E_{x x}=0.4$ without noise. c: Deformed image of $E_{x x}=0.4$ with noise. d: Deformed image of $E_{x y}=0.2$ without noise. e: Deformed image of $E_{x y}=0.2$ with noise.

\subsection{Imaging of Human Lower Leg}

The bones of the lower leg were segmented and excluded from the strain map computation. We processed only soft tissue of the leg. A circular band-pass filter with radius of $1.56 \mathrm{~mm}$ was used to extract the off-center harmonic peak. The calculated harmonic phase image of one direction is shown in Fig. 4(c). We observed from Fig. 
4(a) and Fig. 4(c) that the tag lines correspond well with the wrapped harmonic phase lines. Since the lower leg was indented from the top and around the middle region of the leg, the left and right regions of the lower leg were expectedly having negative and positive displacement values respectively in the $x$ direction (Fig. 5(a)). In the $y$ direction, area located near the indenter has larger displacement (Fig. 5(b)). Since the lower leg was compressed, negative strain values were expected to dominate in both directions. In $y$ direction, smaller strain values were shown in areas away from the indenter and behind the bones (Fig. 5(d)). Due to the presence of noise, strain variation was observed in the bottom left and top right regions.

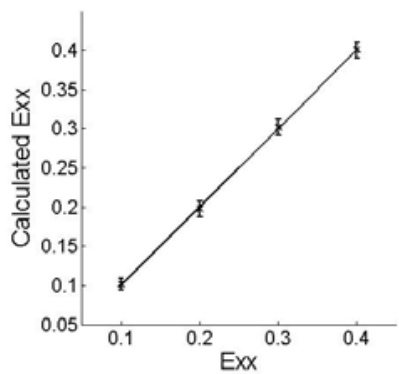

a

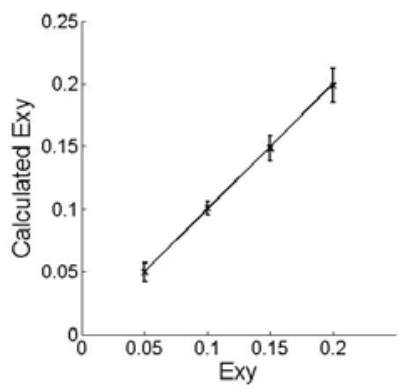

c

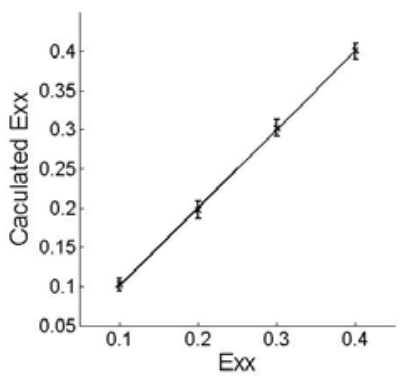

$\mathrm{b}$

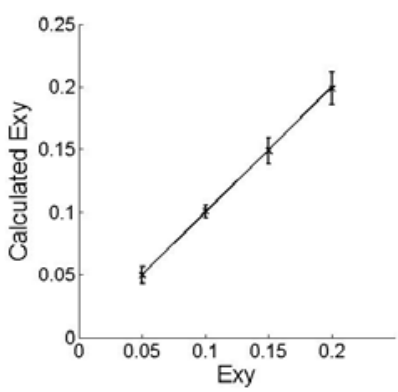

d

Fig. 3. Strain calculation with simulated images. a: Calculated strain of $E_{x x}$ without noise. b: Calculated strain of $E_{x x}$ with noise. c: Calculated strain of $E_{x y}$ without noise. d: Calculated strain of $E_{x y}$ with noise.

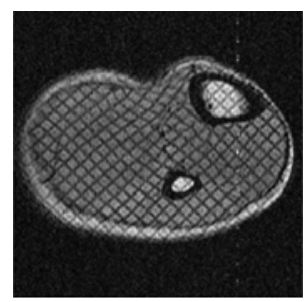

a

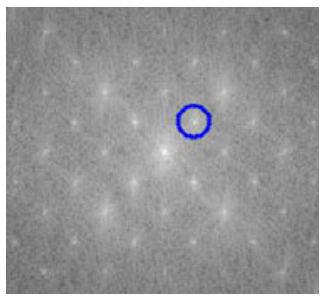

b

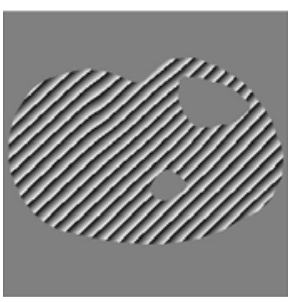

C

Fig. 4. a: Frame 7 of tagging image, b: Band-pass filter in Fourier domain, c: Wrapped harmonic phase image in one direction 


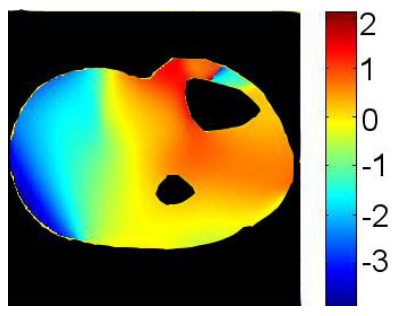

a

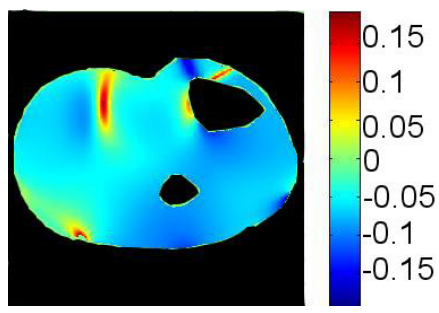

C

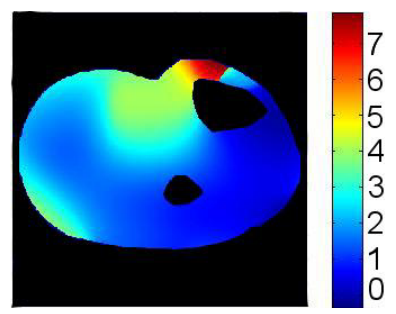

b

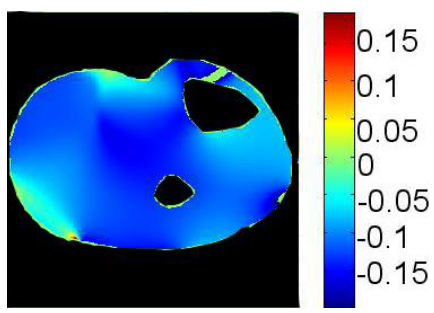

d

Fig. 5. a: Displacement in the $x$ direction (unit: $\mathrm{mm}$ ), b: Displacement in the $y$ direction (unit: $\mathrm{mm}), \mathrm{c}$ : Lagrangian strain in the $x$ direction, $\mathrm{d}$ : Lagrangian strain in the $y$ direction

\section{Discussion and Conclusion}

The proposed imaging system is a clinically viable image acquisition method for quasi-static MRE. To date, most MR strain imaging method for quasi-static MRE requires extensive programming of imaging sequence $[5,22]$ which can be challenging. In contrast, Tagged MRI is an imaging method widely equipped in almost every clinical MRI scanner. Using tagged MRI and the proposed method, strain imaging can be performed without imaging sequence programming. In this paper, tagging images of human lower leg indentation was obtained by building a MR-compatible actuator. Indentation force is recorded at the same time. Motion tracking and strain map of a human leg are calculated using the HARP-based method.

Motion tracking is performed by first measuring the phase shift of soft tissue during indentation. Displacement distribution maps were generated and analyzed. One limitation of the proposed motion tracking method is that it only applies to situation where smooth deformation presents. Validation of the HARP strain calculation is performed on a series of simulated tagged images. Strong agreement between the calculated and true strains is observed. The good noise reduction performance is due to the band-pass filter which also serves as a noise filter. The radius of the band-pass filter is important. Large radius may introduce unnecessary noise while small radius may suppress the actual deformation signal. Optimal radius selection is proposed in [20].

Although the current study was performed on human lower leg, our actuation system can be readily applied to other parts such as abdominal organs, breasts and foot. HARP analysis of images of these organs can be performed to obtain displacement distribution and strain maps. The elasticity distribution of these organs can then be calculated using various elasticity reconstruction methods. 
Acknowledgement. This research is partially supported by NUS-JSPS Joint Research Project Grant.

\section{References}

1. Srinivasan, S., Krouskop, T., Ophir, J.: A quantitative comparison of modulus images obtained using nano indentation with strain elastograms. Ultrasound Med. Biol. 30(7), 899-918 (2004)

2. Ingolf, S., Johannes, B., Jurgen, B.: Analysis of wave patterns in MR elastography of skeletal muscle using coupled harmonic oscillator simulations. Magn. Reson. Imaging 20, 95-104 (2002)

3. Hamhaber, U., Sack, I., Papazoglou, S., Rump, J., Klatt, D., Braun, J.: Three-dimensional analysis of shear wave propagation observed by in vivo magnetic resonance elastography of the brain. Acta Biomater 3, 127-137 (2007)

4. Scott, A.K., Gregory, H.R., Kevin, J.G., Armando, M., Joel, P.F., Clifford, R.J., Richard, L.E.: Magnetic resonance elastography of the brain. Neuro. Image 39, 231-237 (2008)

5. Steele, D.D., Chenevert, T.L., Skovoroda, A.R., Emelianov, S.Y.: Three-dimensional static displacement, stimulated echo NMR elasticity imaging. Phys. Med. Biol. 45, 16331648 (2000)

6. Donald, B.P., Jonathan, B., Abbas, S., Justin, S.: Visualization and quantification of breast cancer biomechanical properties with magnetic resonance elastography, Phys. Med. Biol. 45, 1591-1610 (2000)

7. Atalar, E., McVeigh, E.: Optimization of tag thickness for measuring position with magnetic resonance imaging. IEEE Trans. Med. Imag. 13(1), 152-160 (1994)

8. Guttman, M.A., Prince, J.L., McVeigh, E.R.: Tag and contour detection in tagged MR images of the left ventricle. IEEE Trans. Med. Imaging 13(1), 74-88 (1994)

9. Kerwin, W.S., Prince, J.L.: Tracking MR tag surfaces using a spatiotemporal filter and interpolator. Int. J. Imag. Sys. Tech. 10(2), 128-142 (1999)

10. Deng, X., Thomas, S.D.: Combined tag tracking and strain reconstruction from tagged cardiac MR images without user-defined myocardial contours. J. Magn. Reson. Imaging 21(1), 12-22 (2005)

11. Kumar, S., Goldgof, D.: Automatic tracking of SPAMM grid and the estimation of deformation parameters from cardiac MR images. IEEE Trans. Med. Imag. 13, 122-132 (1993)

12. Amini, A.A., Chen, Y., Curwen, R.W., Mani, V., Sun, J.: Coupled Bsnake grids and constrained thin-plate splines for analysis of 2-D tissue deformations from tagged MRI. IEEE Trans. Med. Imag. 17(3), 344-356 (1998)

13. Qian, Z., Huang, X., Metaxas, D., Axel, L.: Robust segmentation of 4D cardiac MRItagged images via spatio-temporal propagation. In: Proc. SPIE Med. Imag., pp. 580-591 (2005)

14. Prince, J.L., McVeigh, E.R.: Motion estimation from tagged MR image sequences. IEEE Trans. Med. Imag. 11(2), 238-249 (1992)

15. Denney, J.T., Prince, J.L.: Optimal brightness functions for optical flow estimation of deformable motion. IEEE Trans. Image Proc. 3(2), 178-191 (1994)

16. Gupta, S.N., Prince, J.L.: On variable brightness optical flow for tagged MRI. In: Bizais, Y., Barillot, C., DiPaola, R. (eds.) Proc. Inf. Process. Med. Imag., pp. 323-334 (1995)

17. Haber, I., Kikinis, R., Westin, C.-F.: Phase-driven finite element model for spatiotemporal tracking in cardiac tagged MRI. In: Niessen, W.J., Viergever, M.A. (eds.) MICCAI 2001. LNCS, vol. 2208, pp. 1332-1335. Springer, Heidelberg (2001) 
18. Osman, N.F., Kerwin, W.S., McVeigh, E.R., Prince, J.L.: Cardiac motion tracking using cine harmonic phase (harp) magnetic resonance imaging. Magn. Reson. Med. 42, 10481060 (1999)

19. Zhang, S., Douglas, M., Yaroslavsky, L., Summers, R., Dilsizen, V., Fananapazir, L., Bacharach, S.: A Fourier based algorithm for tracking spamm tags in gated magnetic resonance cardiac images. Med. Phys. 23(8), 1359-1369 (1996)

20. Osman, N.F., McVeigh, E.R., Prince, J.L.: Imaging heart motion using harmonic phase MRI. IEEE Trans. Med. Imaging 19, 186-202 (2000)

21. Liu, W., Chen, J.J., Ji, S.B., Allen, S.J., Bayly, P.V., Wickline, S.A., Yu, X.: Harmonic Phase MR Tagging for Direct Quantification of Lagrangian Strain in Rat Hearts after Myocardial Infarction. Magn. Reson. Med. 52, 1282-1290 (2004)

22. Chenevert, T.L., Skovoroda, A.R., O’Donnell, M., Emelianov, S.Y.: Elasticity Reconstructive Imaging by Means of Stimulated Echo MRI. Magn. Reson. Med. 39, 482490 (1998)

23. Pan, L., Prince, J.L., Lima, J.A., Osman, N.F.: Fast tracking of cardiac motion using 3DHARP. IEEE Trans. Biomed. Eng. 52(8), 1425-1435 (2005) 